\title{
Design and implementation of modular home security system with short messaging system
}

\author{
Santoso Budijono ${ }^{1}$, Jeffri Andrianto ${ }^{2}$, Muhammad Axis Novradin Noor ${ }^{3}$ \\ ${ }^{1}$ Subject Content Specialist, Computer Engineering, Bina Nusantara University, Indonesia \\ ${ }^{2}$ Bachelor Computer Engineering, Bina Nusantara University, Indonesia \\ ${ }^{3}$ Bachelor Computer Engineering, Bina Nusantara University, Indonesia
}

\begin{abstract}
Today we are living in $21^{\text {st }}$ century where crime become increasing and everyone wants to secure they asset at their home. In that situation user must have system with advance technology so person do not worry when getting away from his home. It is therefore the purpose of this design to provide home security device, which send fast information to user GSM (Global System for Mobile) mobile device using SMS (Short Messaging System) and also activate - deactivate system by SMS. The Modular design of this Home Security System make expandable their capability by add more sensors on that system. Hardware of this system has been designed using microcontroller AT Mega 328, PIR (Passive Infra Red) motion sensor as the primary sensor for motion detection, camera for capturing images, GSM module for sending and receiving SMS and buzzer for alarm. For software this system using Arduino IDE for Arduino and Putty for testing connection programming in GSM module. This Home Security System can monitor home area that surrounding by PIR sensor and sending SMS, save images capture by camera, and make people panic by turn on the buzzer when trespassing surrounding area that detected by PIR sensor. The Modular Home Security System has been tested and succeed detect human movement.
\end{abstract}

\section{Introduction}

Security is one thing that is very influential in today life, everyone needs security guarantees when they work ${ }^{[1]}$. Like health, security is an important aspect in life. Hence, various kinds of development in the technology field is designed to provide security at all times to protect they assets and privacy. In addition to the course with the application of security system, it can reduce the crime rate in the society especially the crime of theft at home. Due to the increasingly rapid movement of people, making them requires a security technology that has the characteristics of mobile technology in terms of getting information easily and quickly. This Paper mainly focuses on providing security when the user is away from home. SMS (Short Message Service) is a GSM mobile technology that can perform remote communication wherever they are ${ }^{[2]}$. Through this facility messages can send quickly, accurately and at a low cost. Mobile phone with SMS facility will be very useful when applied to integrated security systems, where the information send by a security system and the information received by the user mobile phone in the form of SMS. Another SMS facility are activate - deactivate system using two way information via SMS

Based on this, an integrated security system is made to be able to send information without using a PC (Personal Computer), which helps monitor the situation the house quickly and cheaply ${ }^{[3]}$. C programming language is used in making this system, the program was applied to create a security system works automatically, which can make two way communication with user mobile phone when there is a security breach in the house ${ }^{[4]}$. The two way communication used to turn off the buzzer after active when security breach occur. Modular in design use to make easy expandable for add more sensors to the core system - arduino uno open-source microcontroller platform ${ }^{[5]}$.

\section{Proposed system}

The system contains PIR sensors to detect obstacle, GSM Module for communicate with GSM Phone, Serial Camera for capture picture, SD Card module for save picture. The whole system is controlled by 2 Arduino Uno open-source microcontroller. The system collects all 
information from 2 PIR sensors, process that information and sends SMS to corresponding GSM mobile phone number by using a GSM modem. The use of 2 PIR sensors for more accurate when detect obstacle at area sensors. If PIR sensors detect any obstacle in covered area then the controller capture image through Serial camera and save the captured image to SD Card, after that Arduino controller activate Buzzer alarm and send a SMS to the home owner mobile phone using the GSM Module shown in Figure 1. The system organized in several units like controller, interfacing, gsm module and sensors.

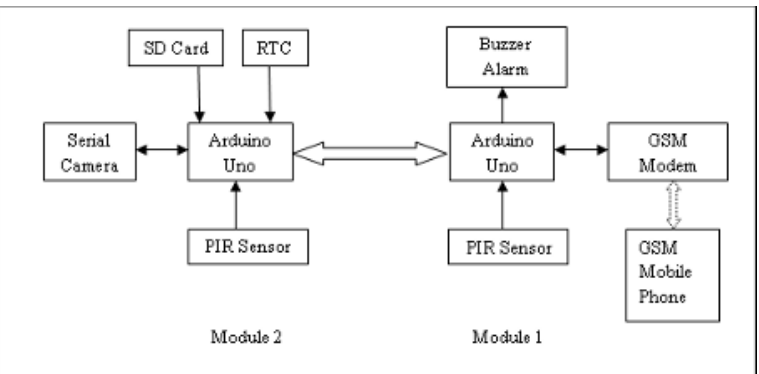

Fig. 1. Home security system block diagram

\section{Hardware}

\subsection{Controller Unit}

The control unit is built using Arduino Uno open-source microcontroller. The Arduino Uno shown in Figure 2 is a microcontroller board based on the ATmega32. It has 14 digital input/output, 6 analog, ceramic resonator, USB connector, power connector, ICSP, and reset button. Support everything needed for the microcontroller; simply connect it to a computer with a USB cable or power it with a AC-to-DC adapter or battery to get started $^{[6]}$.

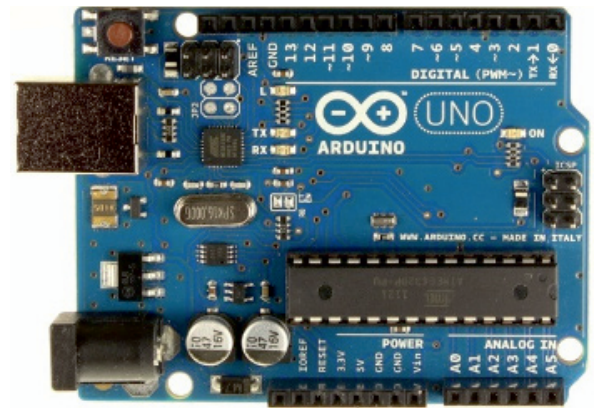

Fig. 2. Arduino uno Open-source Microcontroller

\subsection{GSM modem}

The GSM modem unit is built using SIMCOM SIM900 Modem $^{[7]}$ that specialized for arduino uno controller as shown in Figure 3. This unit can send SMS to user mobile phone and aslo can receive SMS from user.

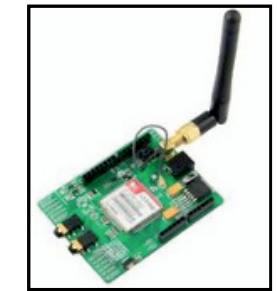

Fig. 3. Simcom modem for Arduino uno controller

\subsection{PIR sensor}

This Passive Infra Red sensor has a potentiometer to calibrate distance and delay time ${ }^{[8]}$. Sensor unit is easy to use, affordable price as shown in Figure 4. This sensor requires $100 \mathrm{uA}-150 \mathrm{uA}$ and voltage conditions $3 \mathrm{Volt}$ 5 Volt to operate, has an accuracy from 0.1 to 6 meters with the ability to work at a temperature of-200C to 700C. other than that, this sensor module also has a working wave length $7 \mathrm{um}-14 \mathrm{um}$ and angle of coverage in 1200 .

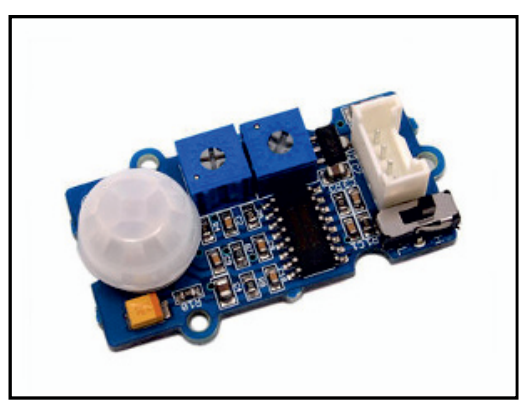

Fig. 4. Passive Infra Red Sensor

\subsection{Buzzer alarm}

This is the DFRobot Buzzer Unit ${ }^{[9]}$. Through the Arduino, this unit will be able to control the buzzer sounds. used in combination with the Arduino board sensors, to achieve the control of an interactive. The module pin definitions: 1 - Output, 2 - Power Supply, 3 ground. Module has interface socket for $\mathrm{PH} 2.0$ as shown in Figure 5. This unit can create sound by arduino controller instruction.

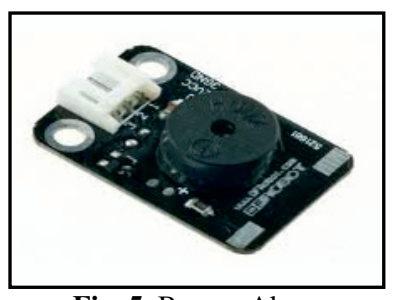

Fig. 5. Buzzer Alarm

\subsection{RTC (Real Time Clock)}

This unit comes fully assembled and pre-programmed with the current time as shown in Figure 6. The included Lithium coin cell battery (CR1225 41mAh) will run the module for a minimum of 9 years (17 years typical) without external 5V power. The DS1307 is accessed via 
the $\mathrm{I} 2 \mathrm{C}$ protocol $^{[\mathbf{1 0 ]}}$. This unit used for make a structured file name for file name image that capture by serial camera.

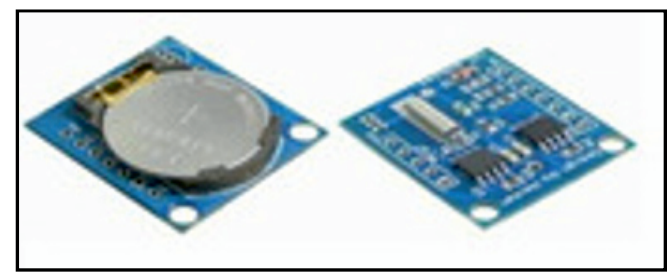

Fig. 6. Real Time Clock

\subsection{Serial Camera}

The Serial Camera as shown in Figure 7 is a JPEG color camera module with DSP image processing to generate $320 * 240$ or $640 * 480$ JPEG image, picture stored in internal buffer and transferred via UART port ${ }^{[11]}$. This unit will capture obstacle area by instruction of arduino controller.

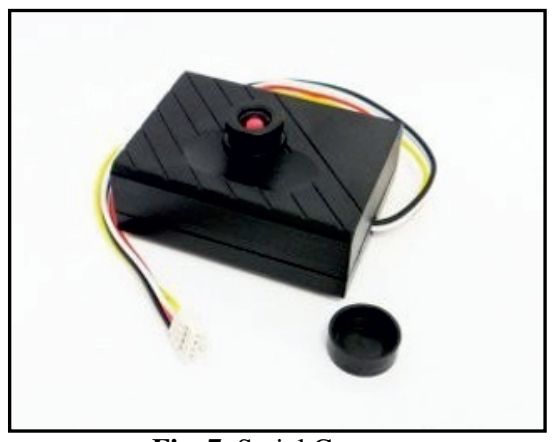

Fig. 7. Serial Camera

\subsection{SD Card}

The unit Arduino SD Card Shield is a simple solution for transferring data to and from a standard SD card ${ }^{[12]}$. The pinout is directly compatible with Arduino as shown if Figure 8.

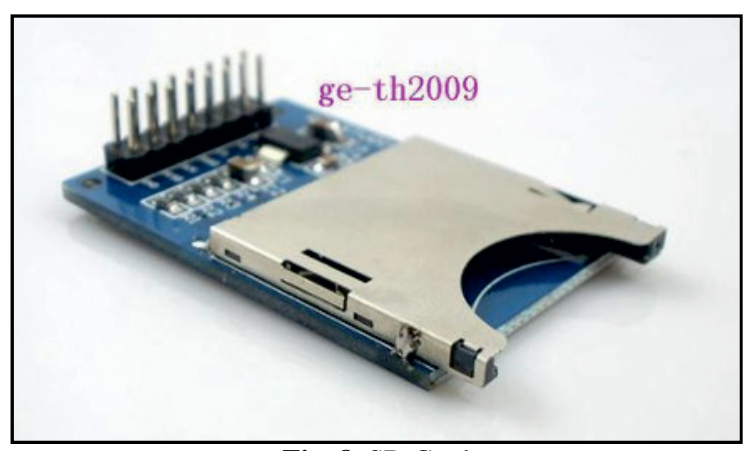

Fig. 8. SD Card

\section{Software}

In this system arduino software from arduino developer is used to develop program for arduino controller. The Arduino Uno can be programmed with the Arduino software (download). Select "Arduino Uno from the Tools > Board menu. The ATmega328 on the Arduino Uno comes with a bootloader that allows you to upload new code to it without the use of an external hardware programmer. It communicates using the STK500 protocol $^{[11]}$.

\section{Flow chart}

Figure 9 and Figure 10 shows the flow chart of the whole system, which also show process of how to turn on or turn off the home security system by sending SMS from user mobile phone. After the home security system on, the system will check the area capture by PIR sensor, if there are obstacles detected system will send notification via SMS to user mobile phone, and also can turn on buzzer alarm.

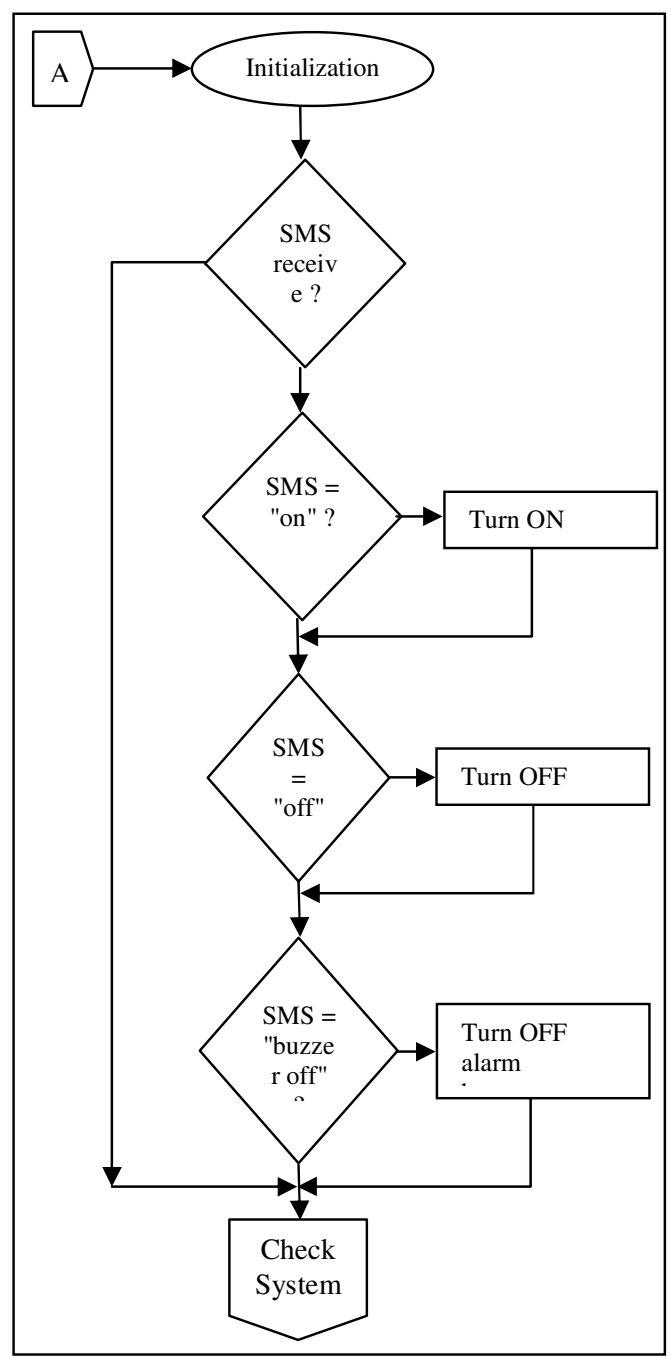

Fig. 9. Flow Chart Module 1 


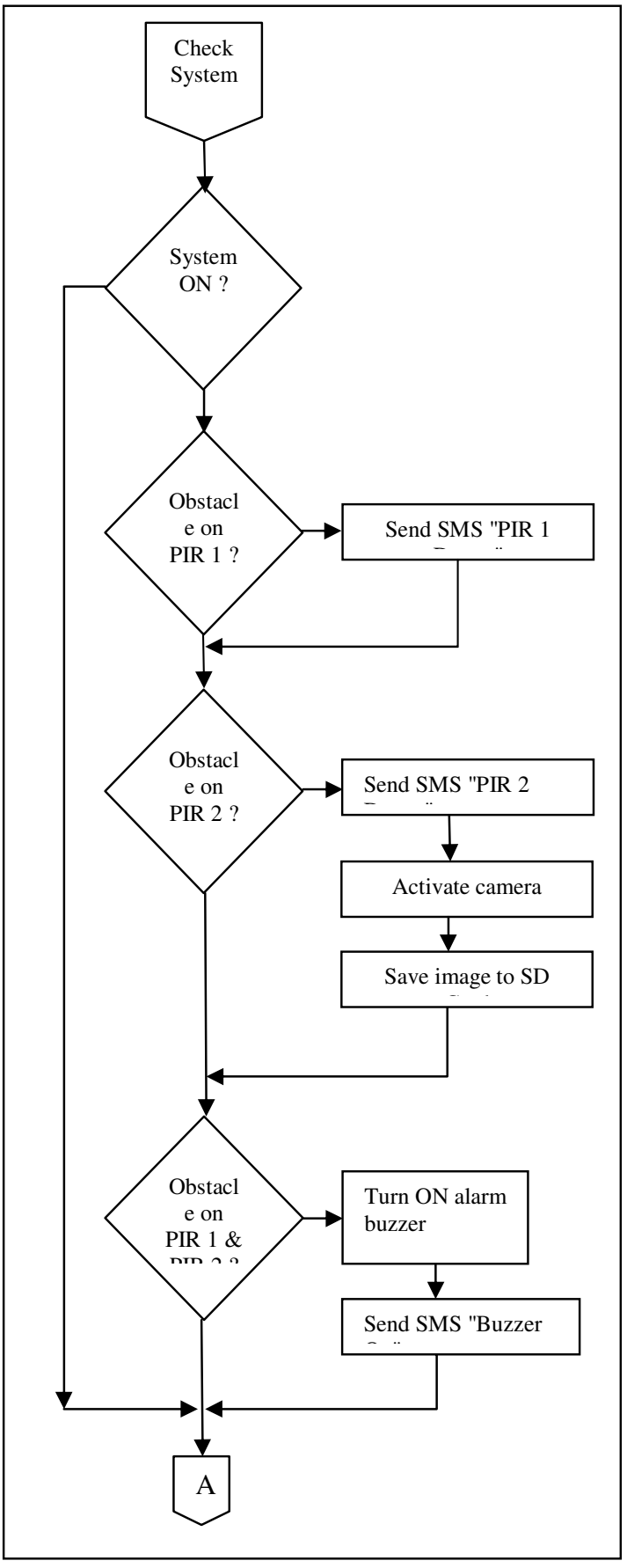

Fig. 10. Flow Chart Module 2

\section{Result}

Figure 11 shows the SMS communication capture on Samsung Galaxy Tab. The system can Send/Receive message To/From Home Security Device via SMS. Message send via SMS by home security device are show on the left side of the figure. Message "PIR 2 detect", "PIR 1 detect" indicate the sensor PIR detect several movement in PIR area. Message "PIR1 \& 2 detect Buzzer Menyala" indicate 2 PIR detect movement in area PIR and buzzer alarm is on. At the right side of the figure indicate message send from Galaxy Tab to home security device via SMS. Message "Of" send to device via SMS for deactivate system and message "On" sent to device via SMS for activate system. Sending message "Buzof" to turn off the buzzer after PIR detect several movement in PIR area. The result of this implementation is good, communication from system security and device (galaxy tab) via sms is working. At the galaxy tab smart phone message displayed if any security attacks capture by PIR and vice versa the galaxy tab smart phone can "off" and "on" the security system via SMS and is receive in good condition by security system

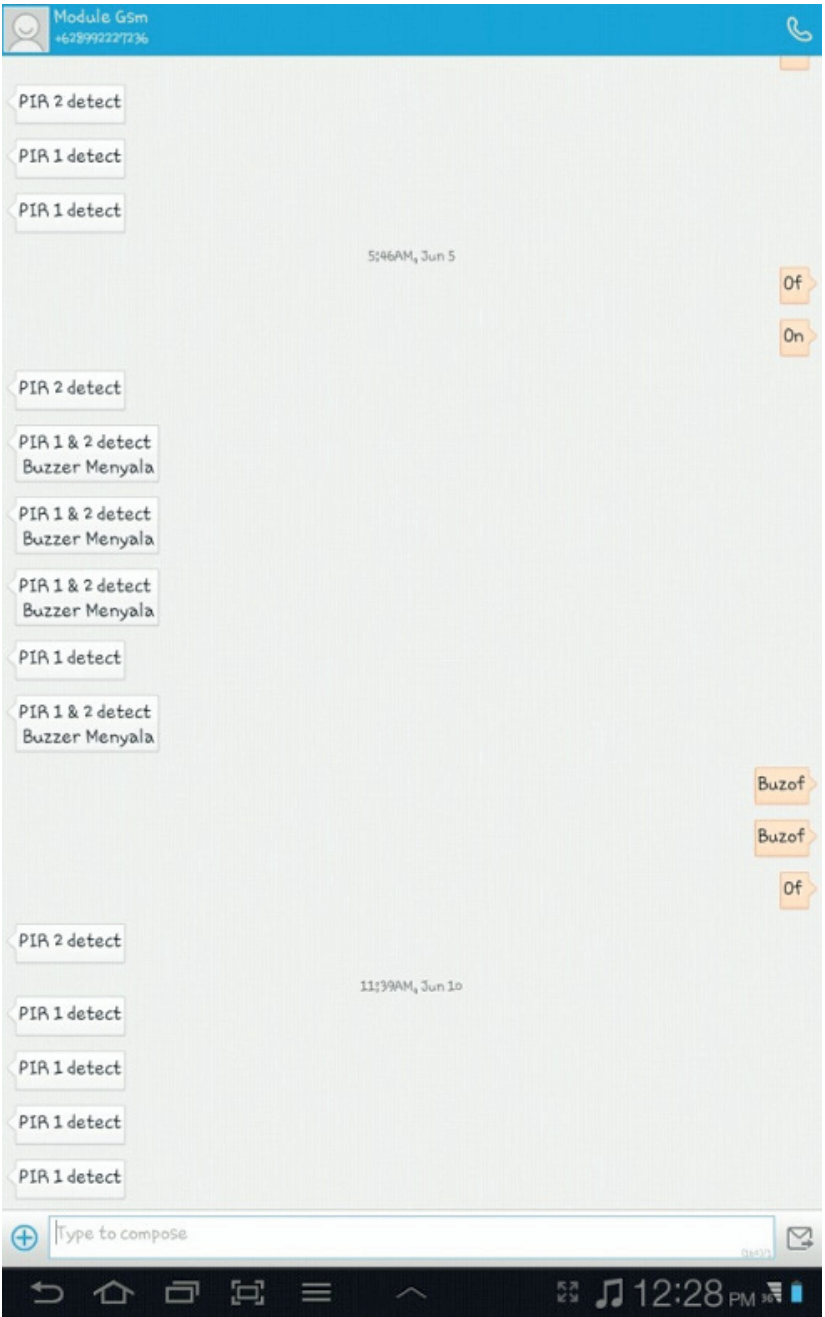

Fig. 11. Result of SMS communication home security system

\section{Conclusion}

This security home feature become draw much attention in the future. People getting more concerned to protect their house from unauthorized people. This system can monitor a house by use of sensors that integrated with a microcontroller and a GSM unit. SMS use to alert users via mobile phone when a possible intrusion occur. Today almost everyone using mobile phone so by use this system user will not have to carry additional device to 
monitor their house. This system is design using modularity to become a flexible system that can be add more sensors without change the whole system, only add some sensors to increase systems functionality. So this system is a modular home security system by using SMS function to communicate between system and user.

\section{References}

1. Sheikh Izzal Azid, Sushi Kumar, Analysis and Performance of a Low Cost SMS Based Home Security System, International Journal of Smart Home, Vol. 5 No. 3, 2011.

2. Sadeque Reza Khan, Ahmed Al Mansur, Alvie Kabir, Shahid Jaman, Nahian Chowdhury, Design and Implementation of Low Cost Home Security System Using GSm network, International Journal of Scientific and Engineering Research, Vol. 3, Issue 3, 2012.

3. Ing. Jan Valouch, Integration of Alarm Systems, International Journal of Disaster Recovery and Business Continuity, Vol. 3, 2012.]

4. Sheikh Izzal Azid, Bibhya Sharma, Intelligent Home: SMS Based Home Security System with Immediate Feedback, World Academy of Science, Engineering and Technology, Vol. 72, 2012.

5. Mahendran.N, Geo Joe Mathai, Veenesh. M.U, Multiple Sensor Feeding Supported Building Automation System Using Arduino Platform With Exposure of 802.15.4 Functionalities, International Journal of Engineering Trends and Technology, Vol. 4 Issue2, 2013.

6. Arduino Uno website, http://arduino.cc/en/Main/arduinoBoardUno

7. Simcom GSM modem website, http://www.simcom.eu/index.php?m=letoltes

8. Seedstudio PIR sensor website, http://www.seeedstudio.com/wiki/File:Twig_-

BISS0001.pdf

9. DFRobot Buzzer Alarm website, http://www.dfrobot.com/wiki/index.php/Digital_Buz zer_Module_(SKU:_DFR0032)

10. DFRobot Realtime clock website, http://www.dfrobot.com/wiki/index.php/Real_Time_ Clock_Module_(DS1307)_(SKU:DFR0151)

11. Twig Serial Camera website, http://seeedstudio.com/wiki/Grove_-_Serial_Camera.

12. MicroSD Adapter for Arduino website, http://www.dfrobot.com/wiki/index.php/MicroSD_ca rd_module_for_Arduino_\%28SKU:DFR0229\%29 